\title{
Transformation Groups for a Schwarzschild-Type Geometry in $f(R)$ Gravity
}

\author{
Emre Dil ${ }^{1}$ and Talha Zafer ${ }^{2}$ \\ ${ }^{1}$ Department of Physics, Sinop University, 57000 Sinop, Turkey \\ ${ }^{2}$ Department of Physics, Sakarya University, 54187 Sakarya, Turkey \\ Correspondence should be addressed to Emre Dil; emredil@sakarya.edu.tr \\ Received 28 June 2016; Revised 17 October 2016; Accepted 23 October 2016 \\ Academic Editor: Sergei D. Odintsov
}

Copyright (C) 2016 E. Dil and T. Zafer. This is an open access article distributed under the Creative Commons Attribution License, which permits unrestricted use, distribution, and reproduction in any medium, provided the original work is properly cited.

\begin{abstract}
We know that the Lorentz transformations are special relativistic coordinate transformations between inertial frames. What happens if we would like to find the coordinate transformations between noninertial reference frames? Noninertial frames are known to be accelerated frames with respect to an inertial frame. Therefore these should be considered in the framework of general relativity or its modified versions. We assume that the inertial frames are flat space-times and noninertial frames are curved space-times; then we investigate the deformation and coordinate transformation groups between a flat space-time and a curved space-time which is curved by a Schwarzschild-type black hole, in the framework of $f(R)$ gravity. We firstly study the deformation transformation groups by relating the metrics of the flat and curved space-times in spherical coordinates; after the deformation transformations we concentrate on the coordinate transformations. Later on, we investigate the same deformation and coordinate transformations in Cartesian coordinates. Finally we obtain two different sets of transformation groups for the spherical and Cartesian coordinates.
\end{abstract}

\section{Introduction}

The coordinate transformations are of great importance in physics, such as nonrelativistic Galilean transformations between flat Euclidean spaces and special relativistic Lorentz transformations between flat Minkowski spacetimes. Another coordinate transformation, the generalized Lorentz transformations of Nelson in noninertial frames in flat space-time, can be viewed as in nongeneral relativistic picture [1]. The related effects and applications of Nelson's generalized Lorentz transformations in locally useful fields can be viewed as Nikolic's works on length contractions in noninertial frames $[2,3]$ and Gron's works $[4,5]$. After these special relativistic, flat space-time coordinate transformations we can consider the general relativistic, curved Riemann space-time coordinate transformations between noninertial frames. While the flat space-time is referred to as the reference frame of an inertial observer, the curved spacetime is referred to as the noninertial observer [6].

In general relativity and similarly in the modified $f(R)$ gravity theory, the noninertial reference frame may be viewed as a gravitationally accelerated object due to the energy-momentum tensor of a massive object. The spacetime curvature is related to the gravitational force of the massive object. Therefore as a source of curvature, a black hole can be taken into account. In this study, we take the spherically symmetric Schwarzschild-type black hole for the source of the curved space-time in which we investigate the deformation and coordinate transformation groups, in the framework of $f(R)$ gravity [7-9].

The modified gravitation theories have become urgent in recent researches, because of the missing matter inconsistency and accelerated expansion of universe. Instead of searching new matter forms of energy to modify the action integral of gravity, $f(R)$ gravity comes into play as a geometrically modified theory by rearranging the Ricci scalar $R$ to a function of the scalar as $f(R)$ in the action integral of the gravity [10].

The extended gravity theories were studied for the problems having complete solutions in general relativity (GR), such as the initial value problem [11], spherically and axially symmetric solutions of black hole, and active galactic nuclei. The solutions of the extended gravity theories should be consistent with the GR. They can be analogous and modified 
solutions of Schwarzschild and Kerr solutions of GR, for example. So the methods for the extended theories should be checked with the solutions of GR [12].

For the spherically symmetric Schwarzschild black hole solutions of GR, we will refer to the spherically symmetric corresponding Schwarzschild-type black hole solutions of Capozziello et al. [13]. Then in this curved and expanded space of Schwarzschild-type black hole, we will study the coordinate transformations from a noninertial frame to an inertial frame being the tangent vector space-time of the Schwarzschild-type space-time, and this flat space-time is represented by the local Lorentz coordinates (LLC). From these transformations we will obtain the related effects, such as length contraction and time dilation, for the $f(R)$ gravity of Schwarzschild-type black hole.

The coordinate transformation between flat tangent vector space-time and the curved space-time of Schwarzschildtype black hole of $f(R)$ gravity will be constructed by the deformation transformations of the flat space-time metric [14-16]. In spherical coordinates this transformation is a conformal transformation between the flat and curved spacetime metrics. Similarly by using the transformations from spherical to Cartesian coordinates, we obtain the metrics of both space-times in Cartesian coordinates, and then we construct the deformation transformation matrices of these two metrics in Cartesian coordinates and obtain the coordinates transformations in Cartesian coordinates for a Schwarzschild-type black hole in $f(R)$ gravity.

Consequently, we firstly find the deformation transformations of metrics in spherical coordinates and the coordinate transformations by using the deformation matrix. Then we find the deformation transformation groups and the coordinate transformation groups in spherical coordinates. Secondly, we convert the metrics in Cartesian coordinates and follow the same procedure within the spherical coordinates. Then we have the deformation transformation and the coordinate transformation groups in Cartesian coordinates for the same flat space-times and curved $f(R)$ gravity Schwarzschild-type black hole space-time.

\section{Transformation Groups in Spherical Coordinates}

Metric tensors of GR and $f(R)$ gravity can be transformed into each other by the conformal rescaling transformation

$$
g_{h r}=\Phi \widetilde{g}_{h r}=f_{R}^{\prime}(R) \widetilde{g}_{h r},
$$

where $g_{h r}$ is the metric tensor in Einstein frame in GR and $\widetilde{g}_{h r}$ is the metric tensor in Jordan frame in $f(R)$ gravity. Here $\Phi=$ $f_{R}^{\prime}(R)$ is the potential related to the expansion of the universe. $f_{R}^{\prime}(R)=\partial f(R) / \partial R$ and $f(R)$ is the modified function of the Ricci scalar in $f(R)$ gravity theory, and $f(R)$ is used in place of the Ricci scalar in Einstein-Hilbert action. The solution of the action integral with $f(R)$ modified Einstein-Hilbert action and the matter Lagrangian action gives the $f(R)$ gravity field equations [17-19].

In $f(R)$ gravity, we can solve the analogous problems for the GR by using modified metric and modified field equations of $f(R)$ gravity. The interested problem here is the spherical symmetric Schwarzschild-type black hole solutions, analogous to GR. We will use Capozziello's approach for the solution $[16,19,20]$. The general solution of this problem is for the constant Ricci Scalar, $R=R_{0}$, the metric of the curved space by the spherical symmetric Schwarzschild-type black hole in the perfect fluid expanding universe with stressenergy tensor $T_{i h}=(\rho+p) u_{i} u_{h}-p g_{i h}$

$$
\begin{aligned}
d s^{2}= & -\left(1+\frac{k_{1}}{r}+\frac{1}{3}(q \chi \rho-\lambda) r^{2}\right) d t^{2} \\
& +\left(1+\frac{k_{1}}{r}+\frac{1}{3}(q \chi \rho-\lambda) r^{2}\right)^{-1} d r^{2}+r^{2} d \theta^{2} \\
& +r^{2} \sin ^{2} \theta d \phi^{2}
\end{aligned}
$$

when $p=-\rho, \lambda=-f\left(R_{0}\right) / 2 f^{\prime}\left(R_{0}\right), \chi=8 \pi G c^{-4}$, and $q^{-1}=f^{\prime}\left(R_{0}\right)$. Also when we look at (1) and compare it with the usual spherical symmetric Schwarzschild black hole metric in GR, we confirm the $g_{00}$ and $g_{11}$ terms are inverse of one another and there is a conformal transformation on GR and $f(R)$ metrics in these terms. The $g_{22}$ and $g_{33}$ terms in $f(R)$ are equal to the ones in GR and the spherical symmetry is seen.

The coordinate transformations between flat tangent vector space-time and curved spherically symmetric Schwarzschild-type black hole space-time in the framework of $f(R)$ gravity can now be investigated. Firstly the deformation matrix between these two diagonal metric tensors of the flat and curved space-time can be found and with the use of this matrix the coordinate transformations are found between these two space-times. Then the deformation transformation is

$$
g_{a b}=\frac{\partial x^{a^{\prime}}}{\partial x^{a}} \frac{\partial x^{b^{\prime}}}{\partial x^{b}} g_{a^{\prime} b^{\prime}}
$$

where the indices run as $a=(t, r, \theta, \phi)$ and $a^{\prime}=\left(t^{\prime}, r^{\prime}, \theta^{\prime}, \phi^{\prime}\right)$ for the flat and curved space-times, respectively. Since the metric tensors are diagonal four by four matrices here, (3) gives the deformation matrix between two metric tensors such as

$$
\begin{aligned}
& \left(\begin{array}{cccc}
g_{t t} & 0 & 0 & 0 \\
0 & g_{r r} & 0 & 0 \\
0 & 0 & g_{\theta \theta} & 0 \\
0 & 0 & 0 & g_{\phi \phi}
\end{array}\right) \\
& =M\left(\begin{array}{cccc}
g_{t^{\prime} t^{\prime}} & 0 & 0 & 0 \\
0 & g_{r^{\prime} r^{\prime}} & 0 & 0 \\
0 & 0 & g_{\theta^{\prime} \theta^{\prime}} & 0 \\
0 & 0 & 0 & g_{\phi^{\prime} \phi^{\prime}}
\end{array}\right),
\end{aligned}
$$




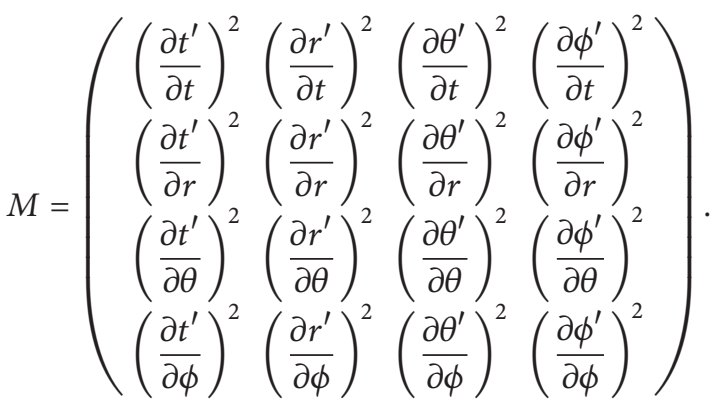

The deformations transformations in (3) are called the conformal transformations of the metrics $[16,21]$. We infer that the coordinate transformation matrix is a diagonal four by four matrix, from (4). The metrics for flat space-time and spherically symmetric Schwarzschild-type black hole spacetime in the framework of $f(R)$ gravity are

$$
\begin{aligned}
& d s^{2}=-d t^{2}+d r^{2}+r^{2} d \theta^{2}+r^{2} \sin ^{2} \theta d \phi^{2}, \\
& d s^{2}=-U d t^{2}+U^{-1} d r^{2}+r^{2} d \theta^{2}+r^{2} \sin ^{2} \theta d \phi^{2},
\end{aligned}
$$

where $U=1+\left(k_{1} / r\right)+(q \chi \rho-\lambda) r^{2} / 3$, respectively $[7-9,13,20]$. For this work in spherical coordinates the nonzero coordinate transformation matrix elements are

$$
\begin{aligned}
& \frac{\partial t^{\prime}}{\partial t}=\sqrt{\left(1+\frac{k_{1}}{r}+\frac{1}{3}(q \chi \rho-\lambda) r^{2}\right)^{-1}}=\sqrt{U^{-1}}, \\
& \frac{\partial r^{\prime}}{\partial r}=\sqrt{\left(1+\frac{k_{1}}{r}+\frac{1}{3}(q \chi \rho-\lambda) r^{2}\right)}=\sqrt{U}, \\
& \frac{\partial \theta^{\prime}}{\partial \theta}=1, \\
& \frac{\partial \phi^{\prime}}{\partial \phi}=1 .
\end{aligned}
$$

Summing all of these, by using the coordinate transformations rule

$$
x^{a^{\prime}}=\frac{\partial x^{a^{\prime}}}{\partial x^{a}} x^{a},
$$

we obtain the coordinate transformations from flat tangent vector space-time to curved space-time of $f(R)$ gravity such as

$$
\begin{aligned}
& t^{\prime}=t \sqrt{\left(1+\frac{k_{1}}{r}+\frac{1}{3}(q \chi \rho-\lambda) r^{2}\right)^{-1}}, \\
& r^{\prime}=r \sqrt{\left(1+\frac{k_{1}}{r}+\frac{1}{3}(q \chi \rho-\lambda) r^{2}\right)}, \\
& \theta^{\prime}=\theta, \\
& \phi^{\prime}=\phi .
\end{aligned}
$$

It is the convenient part for the study of related effects of length contraction and time dilation between inertial and noninertial frames in $f(R)$ Schwarzschild-type black hole curved space-time. So the time dilation and length contraction can be inferred from the solutions of (10) and (11) for point effects. The point effect means that when a time transformation is made between frames, it is assumed to be the space coordinate of the event that is the same, or if a space transformation is made, then the time is assumed to be simultaneous between two events in the frames. The result follows as we get the time dilation equation by using (10)

$$
\Delta t^{\prime}=\sqrt{\left(1+\frac{k_{1}}{r}+\frac{1}{3}(q \chi \rho-\lambda) r^{2}\right)^{-1}} \Delta t
$$

and for length contraction from (11)

$$
\Delta r^{\prime}=\sqrt{\left(1+\frac{k_{1}}{r}+\frac{1}{3}(q \chi \rho-\lambda) r^{2}\right)} \Delta r .
$$

If we call the four by four coordinate transformation matrix for these space-times having these metrics as $\Lambda$, it has the form

$$
\begin{aligned}
\Lambda & =\left(\begin{array}{cccc}
\frac{\partial t^{\prime}}{\partial t} & 0 & 0 & 0 \\
0 & \frac{\partial r^{\prime}}{\partial r} & 0 & 0 \\
0 & 0 & \frac{\partial \theta^{\prime}}{\partial \theta} & 0 \\
0 & 0 & 0 & \frac{\partial \phi^{\prime}}{\partial \phi}
\end{array}\right) \\
& =\left(\begin{array}{cccc}
\sqrt{U^{-1}} & 0 & 0 & 0 \\
0 & \sqrt{U} & 0 & 0 \\
0 & 0 & 1 & 0 \\
0 & 0 & 0 & 1
\end{array}\right) .
\end{aligned}
$$

In this study, we investigate the symmetry group of the coordinate transformation matrix $\Lambda$. The coordinate transformations leave the space-time interval $d s^{2}$ invariant, so the symmetry occurs for these transformations. It is obvious that the determinant of the matrix $\Lambda$ is

$$
\operatorname{det} \Lambda=1 .
$$

Therefore the symmetry group of the matrix $\Lambda$ is found to be SL(4) for the coordinate transformations in spherical coordinates. In addition, the symmetry group of the conformal deformation matrix $M$ is again obtained by the determinant of $M$, such that

$$
M=\left(\begin{array}{cccc}
U^{-1} & 0 & 0 & 0 \\
0 & U & 0 & 0 \\
0 & 0 & 1 & 0 \\
0 & 0 & 0 & 1
\end{array}\right)
$$

Computing the determinant of $M$ obviously gives

$$
\operatorname{det} M=1 \text {. }
$$


We obtain a special linear symmetry group for the four by four conformal deformation matrix $M$. So it also belongs to SL(4) as the matrix $\Lambda$ does. The deformation matrices and consequently the coordinate transformation matrices which can directly be obtained from the deformation matrices belong to general linear group GL(4) that is a general fact [16]. Here these transformation matrices between flat and curved spherically symmetric Schwarzschild-type black hole spacetime in the framework of $f(R)$ gravity are found to be in SL(4) symmetry group.

Now we will investigate the deformation transformation and coordinate transformations in the Cartesian coordinates for the flat space-time and the Schwarzschild-type black hole space-time in the framework of $f(R)$ gravity.

\section{Transformation Groups in Cartesian Coordinates}

It is a common example that, in 2-dimensional Euclidean space, a vector can be transformed as a rotation by an orthogonal matrix belonging to $\mathrm{SO}(2)$ groups. Here we in fact imply that the transformations for the components of the vector are considered to be in Cartesian coordinates. If we consider the components of the transformed vector in the spherical coordinates we obtain a GL(2) transformation matrix for the same event. Therefore for the same events in Section 2, we now investigate the symmetry groups of the deformation transformations and the coordinate transformations in Cartesian coordinates.

For this, we should first transform the metrics from the spherical coordinates to the Cartesian coordinates for the Schwarzschild-type black hole curved space-time. Since the space-time interval is invariant for both spherical and Cartesian coordinates, we use the fact that

$$
g_{\mu^{\prime} \nu^{\prime}}=\frac{\partial x^{a^{\prime}}}{\partial x^{\mu^{\prime}}} \frac{\partial x^{b^{\prime}}}{\partial x^{\nu^{\prime}}} g_{a^{\prime} b^{\prime}}
$$

where $a^{\prime}=\left(t^{\prime}, r^{\prime}, \theta^{\prime}, \phi^{\prime}\right)$ and $\mu^{\prime}=\left(t^{\prime}, x^{\prime}, y^{\prime}, z^{\prime}\right)$. We need to find the elements of the metric tensor in the Cartesian coordinates from (20). For this we will use the elements of the metric tensor in the spherical coordinates from (7). We also need the transformation elements $\partial x^{a^{\prime}} / \partial x^{\mu^{\prime}}$ from spherical to Cartesian coordinate. To find these elements we use $x^{\prime}=$ $r^{\prime} \sin \theta^{\prime} \cos \phi^{\prime}, y^{\prime}=r^{\prime} \sin \theta^{\prime} \sin \phi^{\prime}, z^{\prime}=r^{\prime} \cos \theta^{\prime}$, and $r=$ $\sqrt{x^{\prime 2}+y^{\prime 2}+z^{\prime 2}}$. After a little calculus, we obtain the elements of the metric tensor in Cartesian coordinates, but in terms of the spherical coordinates.

$$
\begin{aligned}
g_{t^{\prime} t^{\prime}}= & -\left(1+\frac{k_{1}}{r}+\frac{1}{3}(q \chi \rho-\lambda) r^{2}\right)=-U, \\
g_{x^{\prime} x^{\prime}}= & \left(\sin \theta^{\prime} \cos \phi^{\prime}\right)^{2} U^{-1}+\left(\cos \theta^{\prime} \cos \phi^{\prime}\right)^{2} \\
& +\left(\sin \phi^{\prime}\right)^{2}, \\
g_{y^{\prime} y^{\prime}}= & \left(\sin \theta^{\prime} \sin \phi^{\prime}\right)^{2} U^{-1}+\left(\cos \theta^{\prime} \sin \phi^{\prime}\right)^{2}
\end{aligned}
$$

$$
\begin{aligned}
& +\left(\cos \phi^{\prime}\right)^{2}, \\
g_{z^{\prime} z^{\prime}}= & \left(\cos \theta^{\prime}\right)^{2} U^{-1}+\left(\sin \theta^{\prime}\right)^{2}, \\
g_{x^{\prime} y^{\prime}}= & g_{y^{\prime} x^{\prime}} \\
= & {\left[\left(\sin \theta^{\prime}\right)^{2} U^{-1}+\left(\cos \theta^{\prime}\right)^{2}-1\right] \cos \phi^{\prime} \sin \phi^{\prime}, } \\
g_{z^{\prime} x^{\prime}}= & g_{x^{\prime} z^{\prime}} \\
= & \left(\sin \theta^{\prime} \cos \phi^{\prime}\right) \cos \theta^{\prime} U^{-1} \\
& +\left(\cos \theta^{\prime} \cos \phi^{\prime}\right)\left(-\sin \theta^{\prime}\right), \\
g_{z^{\prime} y^{\prime}=} & g_{y^{\prime} z^{\prime}} \\
= & \left(\sin \theta^{\prime} \sin \phi^{\prime}\right) \cos \theta^{\prime} U^{-1} \\
& +\left(\cos \theta^{\prime} \sin \phi^{\prime}\right)\left(-\sin \theta^{\prime}\right) .
\end{aligned}
$$

Now we should use the Cartesian coordinates for all spherical coordinates in the elements of the metric, such that

$$
\begin{aligned}
& g_{t^{\prime} t^{\prime}}=-U, \\
& g_{x^{\prime} x^{\prime}}=\frac{x^{\prime 2}}{\left(x^{\prime 2}+y^{\prime 2}+z^{\prime 2}\right)} U^{-1} \\
& +\frac{x^{\prime 2} z^{\prime 2}}{\left(x^{\prime 2}+y^{\prime 2}+z^{\prime 2}\right)\left(x^{\prime 2}+y^{\prime 2}\right)}+\frac{y^{\prime 2}}{\left(x^{\prime 2}+y^{\prime 2}\right)}, \\
& g_{y^{\prime} y^{\prime}}=\frac{y^{\prime 2}}{\left(x^{\prime 2}+y^{\prime 2}+z^{\prime 2}\right)} U^{-1} \\
& +\frac{y^{\prime 2} z^{\prime 2}}{\left(x^{\prime 2}+y^{\prime 2}+z^{\prime 2}\right)\left(x^{\prime 2}+y^{\prime 2}\right)}+\frac{x^{\prime 2}}{\left(x^{\prime 2}+y^{\prime 2}\right)}, \\
& g_{z^{\prime} z^{\prime}}=\frac{z^{\prime 2}}{\left(x^{\prime 2}+y^{\prime 2}+z^{\prime 2}\right)} U^{-1}+\frac{x^{\prime 2}+y^{\prime 2}}{\left(x^{\prime 2}+y^{\prime 2}+z^{\prime 2}\right)}, \\
& g_{x^{\prime} y^{\prime}}=g_{y^{\prime} x^{\prime}} \\
& =\frac{x^{\prime} y^{\prime}}{\left(x^{\prime 2}+y^{\prime 2}+z^{\prime 2}\right)} U^{-1} \\
& +\frac{x^{\prime} y^{\prime} z^{\prime 2}}{\left(x^{\prime 2}+y^{\prime 2}+z^{\prime 2}\right)\left(x^{\prime 2}+y^{\prime 2}\right)}-\frac{x^{\prime} y^{\prime}}{\left(x^{\prime 2}+y^{\prime 2}\right)}, \\
& g_{x^{\prime} z^{\prime}}=g_{z^{\prime} x^{\prime}} \\
& =\frac{x^{\prime} z^{\prime}}{\left(x^{\prime 2}+y^{\prime 2}+z^{\prime 2}\right)} U^{-1}-\frac{x^{\prime} z^{\prime}}{\left(x^{\prime 2}+y^{\prime 2}+z^{\prime 2}\right)}, \\
& g_{y^{\prime} z^{\prime}}=g_{z^{\prime} y^{\prime}} \\
& =\frac{y^{\prime} z^{\prime}}{\left(x^{\prime 2}+y^{\prime 2}+z^{\prime 2}\right)} U^{-1}-\frac{y^{\prime} z^{\prime}}{\left(x^{\prime 2}+y^{\prime 2}+z^{\prime 2}\right)} \text {. }
\end{aligned}
$$


Finally we obtain the elements of the metric tensor for the curved space-time of Schwarzschild-type black hole, in terms of the Cartesian coordinates. The indices for this metric were shown by the primed Greek letters. Since we investigate the transformations between flat and curved space-times in Cartesian coordinates, we use the flat Cartesian metric $g_{\mu \nu}$ whose indices are shown by the unprimed Greek letters such that

$$
g_{\mu \nu}=\left(\begin{array}{cccc}
-1 & 0 & 0 & 0 \\
0 & 1 & 0 & 0 \\
0 & 0 & 1 & 0 \\
0 & 0 & 0 & 1
\end{array}\right)
$$

Then the deformation transformations between the metrics $g_{\mu \nu}$ and $g_{\mu^{\prime} \nu^{\prime}}$ are

$$
g_{\mu \nu}=\frac{\partial x^{\mu^{\prime}}}{\partial x^{\mu}} \frac{\partial x^{\nu^{\prime}}}{\partial x^{\nu}} g_{\mu^{\prime} \nu^{\prime}}
$$

We cannot call the deformation transformations in (30) conformal transformations of the metrics, because both of the metrics are not in diagonal form. As seen in (26)-(28), the curved space-time metric has nondiagonal terms. Now the deformation matrix can be constructed by arranging (30) as below

$$
\begin{aligned}
& \left(\begin{array}{cccc}
g_{t t} & 0 & 0 & 0 \\
0 & g_{x x} & 0 & 0 \\
0 & 0 & g_{y y} & 0 \\
0 & 0 & 0 & g_{z z}
\end{array}\right) \\
& =M\left(\begin{array}{cccc}
g_{t^{\prime} t^{\prime}} & 0 & 0 & 0 \\
0 & g_{x^{\prime} x^{\prime}} & g_{x^{\prime} y^{\prime}} & g_{x^{\prime} z^{\prime}} \\
0 & g_{y^{\prime} x^{\prime}} & g_{y^{\prime} y^{\prime}} & g_{y^{\prime} z^{\prime}} \\
0 & g_{z^{\prime} x^{\prime}} & g_{z^{\prime} y^{\prime}} & g_{z^{\prime} z^{\prime}}
\end{array}\right)
\end{aligned}
$$

and only nonzero elements of the matrix $M$ forms as

$$
M=\left(\begin{array}{cccc}
A & 0 & 0 & 0 \\
0 & B & E & F \\
0 & E & C & G \\
0 & F & G & D
\end{array}\right)
$$

To find the deformation matrix $M$ from (31) is not so easy. Instead of that, we write the inverse transformations in (31) and obtain the inverse deformation matrix $M^{-1}$ in an easier manner. Then we find the matrix $M$ by evaluating the inverse of the matrix $M^{-1}$. Consequently, the matrix $M$ is found to have the elements below

$$
\begin{aligned}
& A=-\frac{1}{g_{t^{\prime} t^{\prime}}}, \\
& B=\frac{-g_{y^{\prime} y^{\prime}} g_{z^{\prime} z^{\prime}}+g_{y^{\prime} z^{\prime}}{ }^{2}}{-g_{x^{\prime} x^{\prime}} g_{y^{\prime} y^{\prime}} g_{z^{\prime} z^{\prime}}-2 g_{x^{\prime} y^{\prime}} g_{y^{\prime} z^{\prime}} g_{x^{\prime} z^{\prime}}+g_{x^{\prime} x^{\prime}} g_{y^{\prime} z^{\prime}}{ }^{2}+g_{z^{\prime} z^{\prime}} g_{x^{\prime} y^{\prime}}{ }^{2}+g_{y^{\prime} y^{\prime}} g_{x^{\prime} z^{\prime}}{ }^{2}}, \\
& C=\frac{-g_{x^{\prime} x^{\prime}} g_{z^{\prime} z^{\prime}}+g_{x^{\prime} z^{\prime}}{ }^{2}}{-g_{x^{\prime} x^{\prime}} g_{y^{\prime} y^{\prime}} g_{z^{\prime} z^{\prime}}-2 g_{x^{\prime} y^{\prime}} g_{y^{\prime} z^{\prime}} g_{x^{\prime} z^{\prime}}+g_{x^{\prime} x^{\prime}} g_{y^{\prime} z^{\prime}}{ }^{2}+g_{z^{\prime} z^{\prime}} g_{x^{\prime} y^{\prime}}{ }^{2}+g_{y^{\prime} y^{\prime}} g_{x^{\prime} z^{\prime}}{ }^{2}}, \\
& D=\frac{-g_{x^{\prime} x^{\prime}} g_{y^{\prime} y^{\prime}}+g_{x^{\prime} y^{\prime}}{ }^{2}}{-g_{x^{\prime} x^{\prime}} g_{y^{\prime} y^{\prime}} g_{z^{\prime} z^{\prime}}-2 g_{x^{\prime} y^{\prime}} g_{y^{\prime} z^{\prime}} g_{x^{\prime} z^{\prime}}+g_{x^{\prime} x^{\prime}} g_{y^{\prime} z^{\prime}}{ }^{2}+g_{z^{\prime} z^{\prime}} g_{x^{\prime} y^{\prime}}{ }^{2}+g_{y^{\prime} y^{\prime}} g_{x^{\prime} z^{\prime}}{ }^{2}}, \\
& E=\frac{g_{z^{\prime} z^{\prime}} g_{x^{\prime} y^{\prime}}-g_{y^{\prime} z^{\prime}} g_{x^{\prime} z^{\prime}}}{-g_{x^{\prime} x^{\prime}} g_{y^{\prime} y^{\prime}} g_{z^{\prime} z^{\prime}}-2 g_{x^{\prime} y^{\prime}} g_{y^{\prime} z^{\prime}} g_{x^{\prime} z^{\prime}}+g_{x^{\prime} x^{\prime}} g_{y^{\prime} z^{\prime}}{ }^{2}+g_{z^{\prime} z^{\prime}} g_{x^{\prime} y^{\prime}}{ }^{2}+g_{y^{\prime} y^{\prime}} g_{x^{\prime} z^{\prime}}{ }^{2}}, \\
& F=\frac{g_{y^{\prime} y^{\prime}} g_{x^{\prime} z^{\prime}}-g_{x^{\prime} y^{\prime}} g_{y^{\prime} z^{\prime}}}{-g_{x^{\prime} x^{\prime}} g_{y^{\prime} y^{\prime}} g_{z^{\prime} z^{\prime}}-2 g_{x^{\prime} y^{\prime}} g_{y^{\prime} z^{\prime}} g_{x^{\prime} z^{\prime}}+g_{x^{\prime} x^{\prime}} g_{y^{\prime} z^{\prime}}{ }^{2}+g_{z^{\prime} z^{\prime}} g_{x^{\prime} y^{\prime}}{ }^{2}+g_{y^{\prime} y^{\prime}} g_{x^{\prime} z^{\prime}}{ }^{2}}, \\
& G=\frac{g_{x^{\prime} x^{\prime}} g_{y^{\prime} z^{\prime}}-g_{x^{\prime} y^{\prime}} g_{x^{\prime} z^{\prime}}}{-g_{x^{\prime} x^{\prime}} g_{y^{\prime} y^{\prime}} g_{z^{\prime} z^{\prime}}-2 g_{x^{\prime} y^{\prime}} g_{y^{\prime} z^{\prime}} g_{x^{\prime} z^{\prime}}+g_{x^{\prime} x^{\prime}} g_{y^{\prime} z^{\prime}}{ }^{2}+g_{z^{\prime} z^{\prime}} g_{x^{\prime} y^{\prime}}{ }^{2}+g_{y^{\prime} y^{\prime}} g_{x^{\prime} z^{\prime}}{ }^{2}} \text {. }
\end{aligned}
$$

By using (22)-(28) in (33) and making the simplifications for these matrix elements, we finally obtain these elements as follows:

$$
\begin{aligned}
& A=\frac{1}{U}, \\
& B=\frac{U x^{2}+y^{2}+z^{2}}{x^{2}+y^{2}+z^{2}},
\end{aligned}
$$

$$
\begin{aligned}
& C=\frac{U y^{2}+x^{2}+z^{2}}{x^{2}+y^{2}+z^{2}}, \\
& D=\frac{U z^{2}+x^{2}+y^{2}}{x^{2}+y^{2}+z^{2}}, \\
& E=\frac{U x y-x y}{x^{2}+y^{2}+z^{2}},
\end{aligned}
$$




$$
\begin{aligned}
& F=\frac{U x z-x z}{x^{2}+y^{2}+z^{2}}, \\
& G=\frac{U y z-y z}{x^{2}+y^{2}+z^{2}} .
\end{aligned}
$$

These are the elements of the deformation matrix $M$. To find the determinant of matrix $M$, we use (34)-(40) in (32), and then we evaluate the determinant, such as

$$
\operatorname{det} M=1 \text {. }
$$

We understand that our matrix belongs to SL(4), because of the determinant. This is in accordance with the general fact that the symmetry group of the deformation matrices belong to $\mathrm{GL}(4)$.

Now we are to find the coordinate transformations between these two space-times having the deformation transformations given by (30)-(32). We need to find the invertible matrix elements $\partial x^{\mu^{\prime}} / \partial x^{\nu}$ between flat and curved space-times in Cartesian coordinates. Therefore we write the matrix multiplication in (31) explicitly. Then we also write the deformation equations with summation convention in (30) explicitly. We equate the metric elements $g_{\mu \nu}$ in both explicit forms; then we obtain the invertible matrix elements $\partial x^{\mu^{\prime}} / \partial x^{\nu}$. From these elements we construct the coordinate transformation matrix $\Lambda$ directly, as given below

$$
\begin{aligned}
\Lambda & =\left(\begin{array}{cccc}
\frac{\partial t^{\prime}}{\partial t} & 0 & 0 & 0 \\
0 & \frac{\partial x^{\prime}}{\partial x} & \frac{\partial x^{\prime}}{\partial y} & \frac{\partial x^{\prime}}{\partial z} \\
0 & \frac{\partial y^{\prime}}{\partial x} & \frac{\partial y^{\prime}}{\partial y} & \frac{\partial y^{\prime}}{\partial z} \\
0 & \frac{\partial z^{\prime}}{\partial x} & \frac{\partial z^{\prime}}{\partial y} & \frac{\partial z^{\prime}}{\partial z}
\end{array}\right) \\
& =\left(\begin{array}{cccc}
\sqrt{A} & 0 & 0 & 0 \\
0 & \sqrt{B} & \frac{E}{\sqrt{C}} & \frac{F}{\sqrt{D}} \\
0 & \frac{E}{\sqrt{B}} & \sqrt{C} & \frac{G}{\sqrt{D}} \\
0 & \frac{F}{\sqrt{B}} & \frac{G}{\sqrt{C}} & \sqrt{D}
\end{array}\right) .
\end{aligned}
$$

We can write these elements more explicitly by using (34)(40) and obtain

$$
\begin{aligned}
& \frac{\partial t^{\prime}}{\partial t}=\sqrt{\frac{1}{U}}, \\
& \frac{\partial x^{\prime}}{\partial x}=\sqrt{\frac{U x^{2}+y^{2}+z^{2}}{x^{2}+y^{2}+z^{2}}}, \\
& \frac{\partial y^{\prime}}{\partial y}=\sqrt{\frac{x^{2}+U y^{2}+z^{2}}{x^{2}+y^{2}+z^{2}}},
\end{aligned}
$$

$$
\begin{aligned}
& \frac{\partial z^{\prime}}{\partial z}=\sqrt{\frac{x^{2}+y^{2}+U z^{2}}{x^{2}+y^{2}+z^{2}}}, \\
& \frac{\partial x^{\prime}}{\partial y}=\frac{(U x y-x y) /\left(x^{2}+y^{2}+z^{2}\right)}{\sqrt{\left(x^{2}+U y^{2}+z^{2}\right) /\left(x^{2}+y^{2}+z^{2}\right)}}, \\
& \frac{\partial x^{\prime}}{\partial z}=\frac{(U x z-x z) /\left(x^{2}+y^{2}+z^{2}\right)}{\sqrt{\left(x^{2}+y^{2}+U z^{2}\right) /\left(x^{2}+y^{2}+z^{2}\right)}}, \\
& \frac{\partial y^{\prime}}{\partial x}=\frac{(U x y-x y) /\left(x^{2}+y^{2}+z^{2}\right)}{\sqrt{\left(U x^{2}+y^{2}+z^{2}\right) /\left(x^{2}+y^{2}+z^{2}\right)}}, \\
& \frac{\partial y^{\prime}}{\partial z}=\frac{(U y z-y z) /\left(x^{2}+y^{2}+z^{2}\right)}{\sqrt{\left(x^{2}+y^{2}+U z^{2}\right) /\left(x^{2}+y^{2}+z^{2}\right)}}, \\
& \frac{\partial z^{\prime}}{\partial x}=\frac{(U x z-x z) /\left(x^{2}+y^{2}+z^{2}\right)}{\sqrt{\left(U x^{2}+y^{2}+z^{2}\right) /\left(x^{2}+y^{2}+z^{2}\right)}}, \\
& \frac{(U y z-y z) /\left(x^{2}+y^{2}+z^{2}\right)}{\sqrt{\left(x^{2}+U y^{2}+z^{2}\right) /\left(x^{2}+y^{2}+z^{2}\right)}} .
\end{aligned}
$$

When we insert these elements into the coordinate transformation matrix $\Lambda$, we evaluate its determinant as follows:

$\operatorname{det} M$

$$
=\frac{\sqrt{U\left(x^{2}+y^{2}+z^{2}\right)\left(x^{2}+y^{2}+z^{2}\right)\left(x^{2}+y^{2}+z^{2}\right)}}{\sqrt{\left(U x^{2}+y^{2}+z^{2}\right)\left(x^{2}+U y^{2}+z^{2}\right)\left(x^{2}+y^{2}+U z^{2}\right)}}
$$

or

$\operatorname{det} M$

$$
=\frac{\sqrt{U}\left(x^{2}+y^{2}+z^{2}\right)^{3 / 2}}{\sqrt{\left(U x^{2}+y^{2}+z^{2}\right)\left(x^{2}+U y^{2}+z^{2}\right)\left(x^{2}+y^{2}+U z^{2}\right)}} .
$$

We infer from the nonzero determinant of the coordinate transformation matrix $\Lambda$ that it is invertible and it belongs to the GL(4) symmetry group since it is a four by four matrix. This is again in accordance with the general fact about the deformation matrices and their resulting coordinate transformation matrices.

The deformation matrix $M$ and the coordinate transformation matrix $\Lambda$ are shown to belong to SL(4) and GL(4), respectively. In Section 2 for the spherical coordinates case, we found that both matrices belong to SL(4), for the transformations from flat tangent vector space-time to curved Schwarzschild-type black hole space-time in the framework of $f(R)$ gravity. These symmetry groups result from the invariance of the space-time interval $d s^{2}$ under these transformations.

Similarly, one can also find the deformation and coordinate transformation groups between the inertial frames 
and the noninertial frames of $f(R)$ gravity axially symmetric Kerr-Type and charged Reissner-Nordström-Type black holes because their metric tensors have nondiagonal terms as in the case of Cartesian coordinates representations (22)(28) for the metric of Schwarzschild-type black hole curved space-time (31). The metric tensor of a charged and rotating $f(R)$ gravity black hole is given as [22]

$$
\begin{aligned}
d s^{2}= & -\frac{\Delta_{r}}{\rho^{2}}\left[d t-\frac{a \sin ^{2} \theta}{\Xi} d \phi\right]^{2}+\frac{\rho^{2}}{\Delta_{r}} d r^{2}+\frac{\rho^{2}}{\Delta_{\theta}} d \theta^{2} \\
& +\frac{\Delta_{\theta} \sin ^{2} \theta}{\rho^{2}}\left[a d t-\frac{r^{2}+a^{2}}{\Xi} d \phi\right]^{2},
\end{aligned}
$$

where

$$
\begin{aligned}
\Delta_{r} & =\left(r^{2}+a^{2}\right)\left(1+\frac{R_{0}}{12} r^{2}\right)-2 M r+\frac{Q^{2}}{1+f^{\prime}\left(R_{0}\right)}, \\
\Xi & =1-\frac{R_{0}}{12} a^{2}, \\
\Delta_{\theta} & =1-\frac{R_{0}}{12} a^{2} \cos ^{2} \theta .
\end{aligned}
$$

Also, $a$ and $Q$ are the angular momentum and the charge of the $f(R)$ gravity black hole, respectively. If $a=0$, (55) turns to be a Reissner-Nordström-Type black hole in $f(R)$ gravity, whereas if $Q=0$, it gives a Kerr-Type $f(R)$ gravity black hole. We can use metric (55) for both Kerr-Type and ReissnerNordström-Type $f(R)$ gravity black holes and rewrite it as

$$
\begin{aligned}
d s^{2}= & \frac{a^{2} \Delta_{\theta} \sin ^{2} \theta-\Delta_{r}}{\rho^{2}} d t^{2} \\
& +\frac{2 a \sin ^{2} \theta}{\rho^{2} \Xi}\left[\Delta_{r}-\Delta_{\theta}\left(r^{2}+a^{2}\right)\right] d t d \phi \\
& +\frac{\rho^{2}}{\Delta_{r}} d r^{2}+\frac{\rho^{2}}{\Delta_{\theta}} d \theta^{2} \\
& +\frac{\Delta_{\theta} \sin ^{2} \theta\left(r^{2}+a^{2}\right)^{2}-\Delta_{r} a^{2} \sin ^{4} \theta}{\rho^{2}} d \phi^{2},
\end{aligned}
$$

and in matrix form

$$
g_{\mu \nu}=\left(\begin{array}{cccc}
\frac{a^{2} \Delta_{\theta} \sin ^{2} \theta-\Delta_{r}}{\rho^{2}} & 0 & 0 & \frac{2 a \sin ^{2} \theta}{\rho^{2} \Xi}\left[\Delta_{r}-\Delta_{\theta}\left(r^{2}+a^{2}\right)\right] \\
0 & \frac{\rho^{2}}{\Delta_{r}} & 0 & 0 \\
0 & 0 & \frac{\rho^{2}}{\Delta_{\theta}} & 0 \\
\frac{2 a \sin ^{2} \theta}{\rho^{2} \Xi}\left[\Delta_{r}-\Delta_{\theta}\left(r^{2}+a^{2}\right)\right] & 0 & 0 & \frac{\Delta_{\theta} \sin ^{2} \theta\left(r^{2}+a^{2}\right)^{2}-\Delta_{r} a^{2} \sin ^{4} \theta}{\rho^{2}}
\end{array}\right) .
$$

By using the similar steps in (31) and (32), we can obtain the deformation matrix $M$ for the inertial observer and the noninertial observer in the space-time curved by KerrType or Reissner-Nordström-Type $f(R)$ gravity black holes. Accordingly, from the same step in (42), we then obtain the coordinate transformation matrix $\Lambda$ for these observers' frame.

\section{Conclusions}

In this paper, we consider the transformations between a curved and flat space-time being the tangent vector spacetime of the curved space-time, and also the coordinates of this tangent space are called the local Lorentz coordinates. The curvature of the space-time used here results from the Schwarzschild-type black hole whose solution for the metric tensor is obtained in the frameworks of modified $f(R)$ gravity [7-9]. We investigated the symmetry groups of the deformation matrix for the transformations of metrics and then the coordinate transformation matrix obtained by this deformation matrix. The extended and straightforward physical meaning and interpretations of the deformations and conformal transformations can be found in the literature; see $[16,23,24]$.

These deformation and coordinate transformation matrices are the identity matrices for the case $U=1$. This means that the both space-times are same flat space-times. Therefore the transformation matrices for deformation and coordinate transformations must be identity matrix, in both coordinate systems.

When we use $U=1$ in spherical coordinates for the transformation matrices $M$ and $\Lambda$ in (18) and (16)w respectively, we can easily realize that they are the identity matrices. Similarly, if we again use $U=1$ in Cartesian coordinates, we find the matrices $M$ and $\Lambda$ in (32) and (42) are the identity matrices. Namely, for $U=1$, all the nondiagonal elements of $M$ in (38) and (40) vanish. In addition, all the nondiagonal elements of $\Lambda$ in (47) and (52) again vanish for $U=1$. Taking $U=1$ can be viewed as a limiting case for checking the calculations for transformation matrices whose symmetry groups are investigated. 
We infer that the deformation matrices, the coordinate transformation matrices, and their symmetry groups can be investigated for the other type of black hole solutions in the framework of modified $f(R)$ gravity. Also we can consider the present transformation groups for the other type of modified gravitation theories for these various black hole solutions as the candidate studies related to this topic.

\section{Competing Interests}

The authors declare that there is no conflict of interests regarding the publication of this paper.

\section{References}

[1] R. A. Nelson, "Generalized Lorentz transformation for an accelerated, rotating frame of reference," Journal of Mathematical Physics, vol. 28, no. 10, pp. 2379-2383, 1987.

[2] H. Nikolic, "Relativistic contraction of an accelerated rod," American Journal of Physics, vol. 67, no. 11, pp. 1007-1012, 1999.

[3] H. Nikolic, "Relativistic contraction and related effects in noninertial frames," Physical Review A, vol. 61, no. 3, Article ID 032109, 8 pages, 2000.

[4] O. Gron, "Acceleration and weight of extended bodies in the theory of relativity," American Journal of Physics, vol. 45, no. 1, pp. 65-70, 1977.

[5] O. Gron, "Relativictic description of a rotating disk," American Journal of Physics, vol. 43, pp. 869-876, 1975.

[6] R. D'Inverno, Introtucing Einstein's Relativity, Oxford University Press, Oxford, UK, 1992.

[7] S. Capozziello and M. D. Laurentis, "Black holes and stellar structures in $\mathrm{f}(\mathrm{R})$-gravity," https://arxiv.org/abs/1202.0394.

[8] S. Nojiri and S. D. Odintsov, "Unified cosmic history in modified gravity: from $F(R)$ theory to Lorentz non-invariant models," Physics Reports, vol. 505, no. 2-4, pp. 59-144, 2011.

[9] S. Nojiri and S. D. Odintsov, "Introduction to modified gravity and gravitational alternative for dark energy," International Journal of Geometric Methods in Modern Physics, vol. 4, no. 1, pp. 115-145, 2007.

[10] S. Capozziello, "Curvature quintessence," International Journal of Modern Physics D, vol. 11, no. 4, pp. 483-491, 2002.

[11] S. Capozziello and S. Vignolo, "A comment on 'The Cauchy problem of $f(R)$ gravity," Classical and Quantum Gravity, vol. 26, no. 16, Article ID 168001, 2009.

[12] S. Capozziello, A. Stabile, and A. Troisi, "Spherically symmetric solutions in $f(R)$ gravity via the Noether symmetry approach," Classical and Quantum Gravity, vol. 24, no. 8, pp. 2153-2166, 2007.

[13] S. Capozziello, A. Stabile, and A. Troisi, "Spherical symmetry in $f(R)$-gravity," Classical and Quantum Gravity, vol. 25, no. 8, Article ID 085004, 2008.

[14] B. Coll, "A universal law of gravitational deformation for General Relativity," in Proceedings of the Spanish Relativistic Meeting, EREs, Salamanca, Spain, 1998.

[15] B. Coll, J. Llosa, and D. Soler, "Three-dimensional metrics as deformations of a constant curvature metric," General Relativity and Gravitation, vol. 34, no. 2, pp. 269-282, 2002.

[16] S. Capozziello and M. D. Laurentis, "Extended theories of gravity," Physics Reports, vol. 509, no. 4-5, pp. 167-321, 2011.
[17] H. A. Buchdahl, "Monthly notices of the royal astronomical society," Monthly Notices of the Royal Astronomical Society, vol. 150 , no. 1, pp. 1-8, 1970.

[18] A. A. Starobinsky, "A new type of isotropic cosmological models without singularity," Physics Letters B, vol. 91, no. 1, pp. 99-102, 1980.

[19] H. Kleinert and H.-J. Schmidt, "Cosmology with curvaturesaturated gravitational lagrangian $R / \sqrt{1+l^{4} R^{2}}$," General Relativity and Gravitation, vol. 34, no. 8, pp. 1295-1318, 2002.

[20] J. D. Barrow and A. C. Ottewill, "The stability of general relativistic cosmological theory," Journal of Physics A: Mathematical and General, vol. 16, no. 12, pp. 2757-2776, 1983.

[21] M. Carmeli, Group Theory and General Relativity, McGraw-Hill International Book, London, UK, 1977.

[22] A. Larrañaga, "A rotating charged black hole solution in $f(R)$ gravity," Pramana, vol. 78, no. 5, pp. 697-703, 2012.

[23] N. Lanahan-Tremblay and V. Faraoni, "The Cauchy problem of $f(R)$ gravity," Classical and Quantum Gravity, vol. 24, no. 22, pp. 5667-5679, 2007.

[24] G. Allemandi, M. Capone, S. Capozziello, and M. Francaviglia, "Conformal aspects of the Palatini approach in extended theories of gravity," General Relativity and Gravitation, vol. 38, no. 1, pp. 33-60, 2006. 

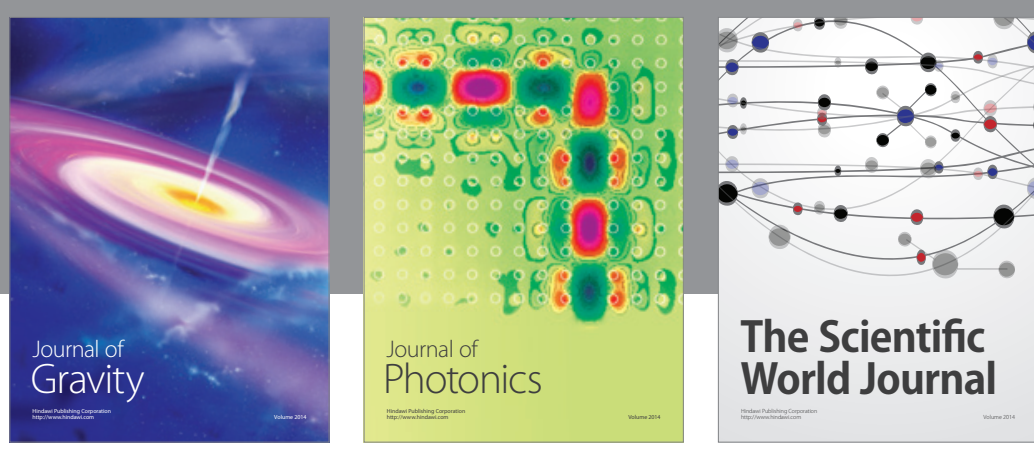

The Scientific World Journal
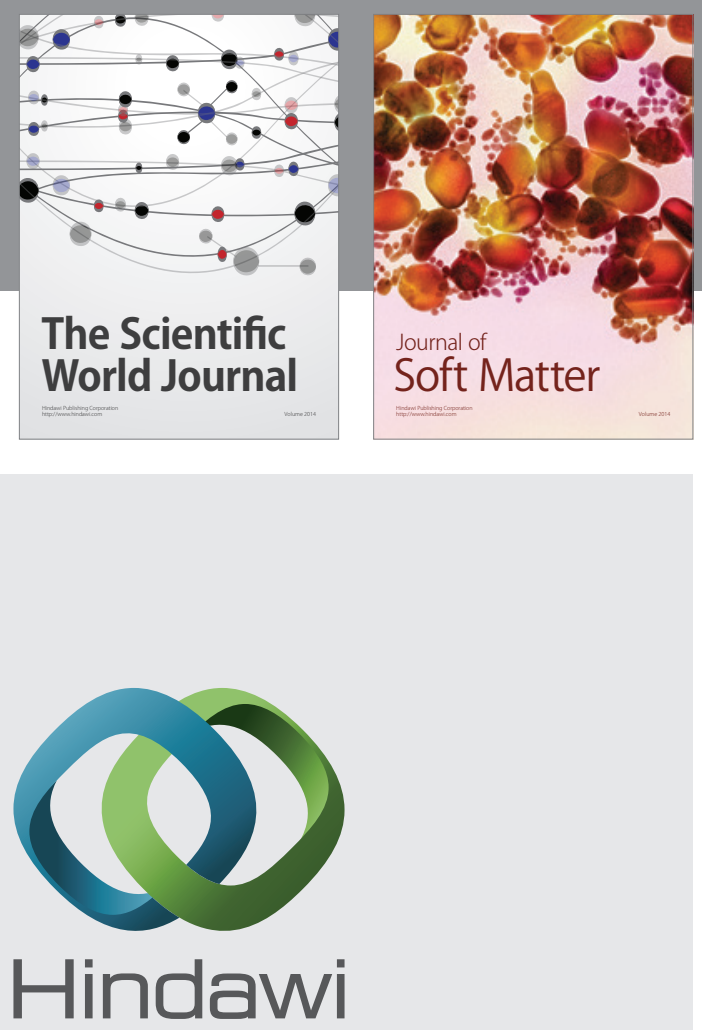

Submit your manuscripts at

http://www.hindawi.com

nternational Journal of

Statistical Mechanics
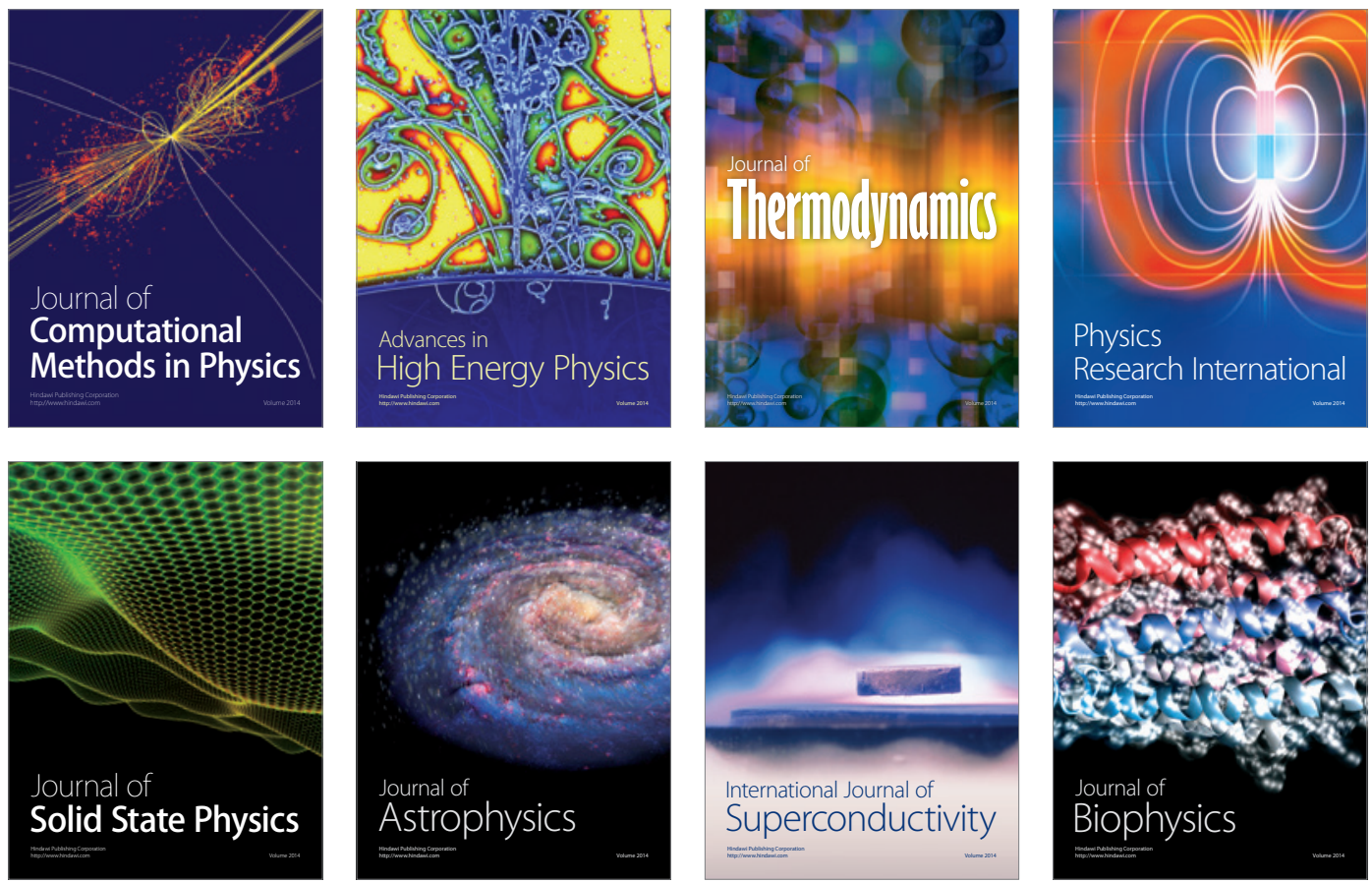
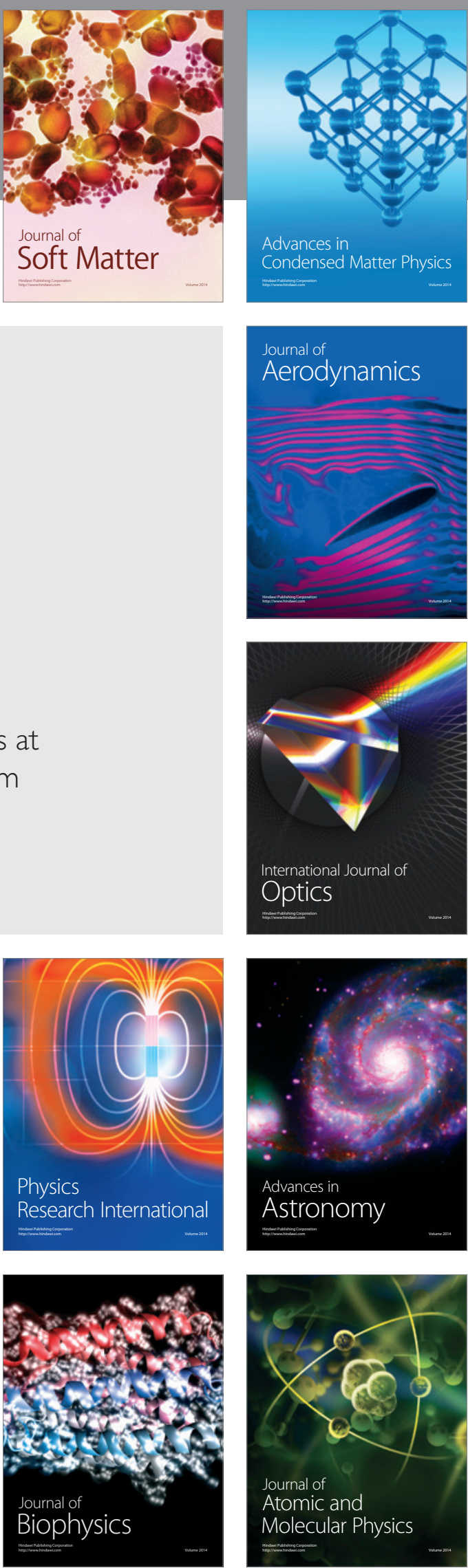